\title{
Difference radical in terms of shifting zero and applications to the Stothers-Mason theorem
}

\author{
Katsuya Ishizaki* and Zhi-Tao Wen ${ }^{\dagger}$
}

\begin{abstract}
In this paper, we study the shifting zeros with its heights and an analogue to difference radical. We focus on the Stothers-Mason theorem by using falling factorials. As applications, we discuss the difference version of the Fermat type functional equations. Some examples are given.
\end{abstract}

Keyword: falling factorials, shifting zeros, difference radical, the Stothers-Mason theorem, Fermat type functional equations

2020MSC: 39B10; 30D35.

\section{Introduction}

Let $P$ be a polynomial. The radical $\operatorname{rad}(P)$ is the product of distinct linear factors of $P$. We study a difference analogue of radical of a complex polynomial, and consider a difference analogue of the Stothers-Mason theorem. As applications, we study the difference version of the Fermat type functional equations, see e.g., 4], [9].

Let $a, b$ and $c$ be relatively prime polynomials such that not all of them are identically zero. The Stothers-Mason theorem [10], [16], see also e.g., [15] states that if they satisfy $a+b=c$, then

$$
\max \{\operatorname{deg}(a), \operatorname{deg}(b), \operatorname{deg}(c)\} \leq \operatorname{deg}(\operatorname{rad}(a b c))-1 .
$$

A difference analogue of the Stothers-Mason theorem is seen in e.g., [4. One of the purpose of this paper is that we give an alternative approach to difference analogues of radical of polynomials in terms of shifting zero different from [4].

We recall basic notations in difference calculus. Given a function $f$, we denote by $\Delta f(z)=f(z+1)-f(z)$ the difference operator. Let $n$ be a nonnegative integer. Define $\Delta^{n} f(z)=\Delta\left(\Delta^{n-1} f(z)\right)$ for $n \geq 1$, and write $\Delta^{0} f=f$. Define $z^{\underline{0}}=1$ and

$$
z^{\underline{n}}=z(z-1) \cdots(z-n+1)=n !\left(\begin{array}{l}
z \\
n
\end{array}\right), \quad n=1,2,3, \ldots,
$$

which is called a falling factorial, see e.g., [1, Pages 6-7], [5, Subsection A.2], [12, Page 25]. This yields $\Delta z^{\underline{n}}=(z+1)^{\underline{n}}-z^{\underline{n}}=n z \underline{n-1}$, which corresponds to $\left(z^{n}\right)^{\prime}=n z^{n-1}$ in the differential calculus.

\footnotetext{
* Supported by JSPS KAKENHI Grant Number 20K03658

$\dagger$ Supported by the National Natural Science Foundation of China (No. 11971288 and No. 11771090) and Shantou University SRFT (NTF18029)
} 
Let $f$ be a complex function and let $n \in \mathbb{N}$. Consider products of the forms $f^{\underline{n}}(z)=$ $f(z) f(z-1) \cdots f(z-n+1), n=1,2,3, \ldots, f^{0}=1$ and $f^{\bar{n}}(z)=f(z) f(z+1) \cdots f(z+n-1)$, $n=1,2,3, \ldots, f^{\overline{0}}=1$. We call $f^{\underline{n}}$ a falling (descending) factorial expression, and call $f^{\bar{n}}$ a raising (ascending) factorial expression, see e.g., [12, Page 25]. The falling factorial $z^{\underline{n}}=z(z-1) \cdots(z-n+1)$ given in (1.2) is the falling factorial expression of $f(z)=z$.

In Section 2, we give the definition of shifting zero and study some properties of it with difference calculus. We discuss the difference radical by using shifting zero and focus on the difference analogue of the Stothers-Mason theorem by using falling factorials in Section 3. Section 4 is concerned with an extension of difference analogue of the StothersMason theorem. We treat in Section 5 polynomial solutions of difference version of the Fermat type functional equations.

\section{Shifting zero}

Let $f$ be analytic in a domain $G \subset \mathbb{C}$ and let $n \in \mathbb{N}$. Suppose that $z_{0}, z_{0}+1, \ldots, z_{0}+n \in G$. The point $z_{0}$ is called a shifting zero of $f(z)$ with height $n$ in $G$ provided $f\left(z_{0}\right)$ and all difference $\Delta^{k} f\left(z_{0}\right)$ vanish for every $0 \leq k<n$, but $\Delta^{n} f\left(z_{0}\right) \neq 0$. In particular, if $z_{0}$ is a shifting zero of $f$ with height 1 , then we also call $z_{0}$ is a shifting zero of $f$ with simple height.

Example 2.1 The function $f(z)=2^{z}=e^{z \ln 2}$ has no zeros, which has no shifting zeros. Let $g$ be defined as $g(z)=z^{2}(z-1)(z-2) 2^{z}$, which can be written

$$
g(z)=z^{\underline{3}} \cdot z 2^{z}=(z-1)^{2} \cdot z^{2} 2^{z}=(z-2)^{\underline{1}} \cdot z^{2}(z-1) 2^{z} .
$$

Then $z=0$ is a shifting zero of $g$ with height 3 in $\mathbb{C}$. In addition, $z=1$ and $z=2$ are the shifting zeros of $g$ with height 2 and 1 in $\mathbb{C}$, respectively.

Consider the Euler Gamma function $\Gamma$. It is known that $1 / \Gamma$ is a transcendental entire function that has simple zeros at $0,-1,-2, \ldots$ This gives that for any $n \in \mathbb{N}, z=-n$ is a shifting zero of $1 / \Gamma$ with height $n+1$ in $\mathbb{C}$, see e.g., [17, Page 236].

Example 2.2 Consider a polynomial $f(z)=z^{2}(z-1)^{3}$. It gives us that $z=0$ is a zero of $f$ with order 2 , and $z=1$ is a zero of $f$ with order 3 . We could also see that $z=0$ is a shifting zero of $f$ with height 2 in $\mathbb{C}$, and $z=1$ is a shifting zero of height 1 in $\mathbb{C}$. In fact, $f$ is of the form

$$
f(z)=\left(z^{\underline{2}}\right)^{2}(z-1)=z^{\underline{2}} \cdot z(z-1)^{2} .
$$

Remark 1 A function $f(z)$ may have a shifting zero with infinite height in $\mathbb{C}$. For example, any integer point is the zero of $f(z)=e^{2 \pi i z}-1$. If a function $f$ has a shifting zero with infinite height, then the order of growth of $f$ is at least 1. It is important to study the order of growth of solutions of nonlinear difference equations. For example, let $w$ be a solution of a difference Riccati equation

$$
w(z+1 / 2)=\frac{A(z)}{w(z)},
$$

where $A(z)$ is a nonconstant rational function. Suppose that all zeros and poles of $A(z)$ are lying on $|z|<R$. Let $z=z_{0}$ be a zero of $w(z)$ such that $\left|z_{0}\right|>R$, it gives $z_{0}+1, z_{0}+2, \ldots$ are zeros of $w(z)$. Then $z_{0}$ is a shifting zero of $w$ with infinite height in $\mathbb{C}$, otherwise, $w$ has at most finitely many zeros and poles. It shows that there does not exist any transcendental meromorphic solutions of (2.1) of order less than 1. 
It is well known that $z=z_{0}$ is a zero of an analytic function $f$ with order $n$ if and only if $f$ is of the form $f=\left(z-z_{0}\right)^{n} g(z)$, where $g(z)$ is analytic at $z=z_{0}$ and $g\left(z_{0}\right) \neq 0$. The difference analogue of this result is given as follows.

Theorem 2.1 Let $f$ be analytic in the domain $G \subset \mathbb{C}$ and let $n \in \mathbb{N}$. Suppose that $z_{0}, z_{0}+1, \ldots, z_{0}+n \in G$. The point $z_{0}$ is a shifting zero of $f(z)$ with height $n$ in $G$ if and only if there exists an analytic function $g(z)$ in $G$ such that

$$
f(z)=\left(z-z_{0}\right)^{\underline{n}} g(z)
$$

and $g\left(z_{0}+n\right) \neq 0$.

Proof. Let $z_{0}$ be a shifting zero of $f$ with height $n$ in $G$. It is known that for any $z \in G$ and $k \in \mathbb{N}$

$$
f(z+k)=\sum_{j=0}^{k}\left(\begin{array}{l}
k \\
j
\end{array}\right) \Delta^{j} f(z) .
$$

Since $\Delta^{j} f\left(z_{0}\right)$ vanish for $0 \leq j \leq k<n$, (2.3) implies that $f\left(z_{0}+k\right)=0$ for $0 \leq k<n$. Further, setting $z=z_{0}$ and $k=n$ in (2.3), we see that $f\left(z_{0}+n\right)=\Delta^{n} f\left(z_{0}\right) \neq 0$. Hence we can write $f(z)$ in the form (2.2) and $g\left(z_{0}+n\right) \neq 0$.

Let us prove another direction as follows. We also have for any $z \in \mathbb{C}$ and $k \in \mathbb{N}$

$$
\Delta^{k} f(z)=\sum_{j=0}^{k}\left(\begin{array}{l}
k \\
j
\end{array}\right)(-1)^{k-j} f(z+j) .
$$

Assume that $f$ is of the form (2.2) and $g\left(z_{0}+n\right) \neq 0$. Then by means of (2.4),$f\left(z_{0}\right)$ and all difference $\Delta^{k} f\left(z_{0}\right)$ vanish for $0<k<n$, but $\Delta^{n} f\left(z_{0}\right) \neq 0$. That proves $z_{0}$ is a shifting zero of $f$ with height $n$ in $G$. For formulas (2.3) and (2.4), see e.g., [6, Page 14], [14, Page 4].

Corollary 2.2 Let $f$ be analytic in the domain $G \subset \mathbb{C}$ and let $n \in \mathbb{N}$. Suppose that $z_{0}, z_{0}+1, \ldots, z_{0}+n \in G$. If $z_{0}$ is a shifting zero of $f$ with height $n$ in $G$, then $z_{0}$ is a shifting zero of $\Delta f$ with height $n-1$.

Proof. Since $z_{0}$ is a shifting zero of $f$ with height $n$ in $G$, there exists an analytic function $g(z)$ in $G$ such that

$$
f(z)=\left(z-z_{0}\right)^{\underline{n}} g(z)
$$

and $g\left(z_{0}+n\right) \neq 0$. Moreover, we have

$$
\begin{aligned}
\Delta f(z) & =\left(z+1-z_{0}\right)^{\underline{n}} g(z+1)-\left(z-z_{0}\right)^{\underline{n}} g(z) \\
& =\left(z-z_{0}\right) \frac{n-1}{n}\left(\left(z+1-z_{0}\right) g(z+1)-\left(z-z_{0}-n+1\right) g(z)\right) \\
& =\left(z-z_{0}\right) \frac{n-1}{n} Q(z) .
\end{aligned}
$$

We see that $Q\left(z_{0}+n-1\right)=n g\left(z_{0}+n\right) \neq 0$. It gives us that $z_{0}$ is a shifting zero of $\Delta f$ with height $n-1$ from Theorem 2.1 .

The next theorem puts the focus on an entire function of the order of growth less than 1, which is a particular case of Theorem 2.1. Let $f$ be an entire function of the order of 
growth $\rho(f)<1$. By means of [5, Theorem 1.1], it follows that $f$ can be written by a binomial (factorial) series in $\mathbb{C}$

$$
f(z)=\sum_{j=0}^{\infty} a_{j}\left(z-z_{0}\right)^{\underline{j}}=a_{0}+a_{1}\left(z-z_{0}\right)^{\underline{1}}+a_{2}\left(z-z_{0}\right)^{\underline{2}}+\cdots+a_{j}\left(z-z_{0}\right)^{\underline{j}}+\cdots,
$$

where the sequence $\left\{a_{j}\right\}$ satisfies

$$
\chi\left(\left\{a_{j}\right\}\right)=\limsup _{j \rightarrow \infty} \frac{j \log j}{-\log \left|a_{j}\right|}<1 .
$$

Theorem 2.3 Let $f$ be an entire function of the order of growth less than 1, and let $n \in \mathbb{N}$. The point $z_{0}$ is a shifting zero of $f(z)$ with height $n$ in $\mathbb{C}$ if and only if there exists an entire function $g$ of the order of growth less than 1 such that

$$
f(z)=\left(z-z_{0}\right)^{\underline{n}} g(z)
$$

and $g\left(z_{0}+n\right) \neq 0$. Furthermore, $f$ and $g$ are represented by binomial series

$$
f(z)=\sum_{j=n}^{\infty} a_{j}\left(z-z_{0}\right)^{\underline{j}} \quad \text { and } \quad g(z)=\sum_{j=0}^{\infty} a_{n+j}\left(z-z_{0}-n\right)^{\underline{j}} .
$$

Proof. We write $f$ as in (2.5). Suppose that $z_{0}$ is a shifting zero with height $n$. We have $\Delta^{k} f\left(z_{0}\right)=0$ for every $0 \leq k \leq n-1$, and $\Delta^{n} f\left(z_{0}\right) \neq 0$. Then $a_{k}=0$ for every $0 \leq k \leq n-1$ and $a_{n} \neq 0$, which implies

$$
\begin{aligned}
f(z)=\sum_{j=n}^{\infty} a_{j}\left(z-z_{0}\right)^{\underline{j}} & =a_{n}\left(z-z_{0}\right)^{\underline{n}}+a_{n+1}\left(z-z_{0}\right)^{\frac{n+1}{}}+a_{n+2}\left(z-z_{0}\right) \frac{n+2}{}+\cdots \\
& =\left(z-z_{0}\right)^{\underline{n}}\left(a_{n}+a_{n+1}\left(z-z_{0}-n\right)^{\underline{1}}+a_{n+2}\left(z-z_{0}-n\right)^{\underline{2}}+\cdots\right) .
\end{aligned}
$$

We set $g$ as

$$
g(z)=\sum_{j=0}^{\infty} a_{n+j}\left(z-z_{0}-n\right)^{\underline{j}}=a_{n}+a_{n+1}\left(z-z_{0}-n\right)^{\underline{1}}+a_{n+2}\left(z-z_{0}-n\right)^{\underline{2}}+\cdots .
$$

Then $g(z)$ is an entire function of the order of growth $\rho(g)<1$ and $g\left(z_{0}+n\right)=a_{n} \neq 0$. Therefore, we proved that $f$ is of the form (2.7).

In what follows, we proceed to prove another direction of this theorem. Let $g$ be an entire function of the order of growth $\rho(g)<1$ such that $g\left(z_{0}+n\right) \neq 0$. Then by [5, Theorem 1.1], $g$ can be supposed as in (2.8) satisfying (2.6). If $f$ is of the form (2.7), then

$$
f(z)=\left(z-z_{0}\right)^{\underline{n}} g(z)=\left(z-z_{0}\right)^{\underline{n}}\left(\sum_{j=0}^{\infty} a_{n+j}\left(z-z_{0}-n\right)^{\underline{j}}\right)=\sum_{j=n}^{\infty} a_{j}\left(z-z_{0}\right)^{\underline{j}},
$$

where $a_{n} \neq 0$. From [5, Theorem 1.1], it gives that $f$ is entire function of the order of growth $\rho(f)<1$. Moreover, it is clear that $\Delta^{k} f\left(z_{0}\right)=0$ for every $0 \leq k \leq n-1$ and $\Delta^{n} f\left(z_{0}\right) \neq 0$. Therefore, $z_{0}$ is a shifting zero of $f$ with height $n$ in $\mathbb{C}$. We thus proved our assertion. 


\section{Difference radical}

Let $P$ be a polynomial with degree $p$, and $z_{1}$ be a shifting zero of $P$ with height $n_{1}$ and $P\left(z_{1}-1\right) \neq 0$. Theorem 2.1 states that there exists a polynomial $P_{1}$ with degree $p-n_{1}$ such that $P(z)=\left(z-z_{1}\right) \frac{n_{1}}{} P_{1}(z)$. Let $z_{2}$ be a shifting zero of $P_{1}$ with height $n_{2}$ and $P\left(z_{2}-1\right) \neq 0$. Then there exists a polynomial $P_{2}$ with degree $p-n_{1}-n_{2}$ such that $P_{1}(z)=\left(z-z_{2}\right) \underline{n_{2}} P_{2}(z)$. Repeating this argument for finitely many times, we see that $P(z)$ can be written uniquely as

$$
P(z)=A \prod_{j=1}^{N}\left(z-z_{j}\right) \frac{n_{j}}{},
$$

where $A$ is a nonzero constant, and $p=n_{1}+\cdots+n_{N}$. Note that it is possible that $z_{j}=z_{k}$ even though $j \neq k$ in (3.1). We define the difference radical $\operatorname{rad}_{\Delta}(P)$ by product of these linear factors, i.e.,

$$
\operatorname{rad}_{\Delta}(P)=\prod_{j=1}^{N}\left(z-z_{j}\right) .
$$

For example, if $g(z)=z^{2}(z-1)(z-2)=z^{\underline{1}} z^{\underline{3}}$, then $\operatorname{rad}_{\Delta}(g)=z^{2}$.

In [4] the definition of $\kappa$-difference radical $\operatorname{rãd}_{\kappa}(P)$ of a polynomial $P$ is given as

$$
\operatorname{rãd}_{\kappa}(P)=\prod_{w \in \mathbb{C}}(z-w)^{d_{\kappa}(w)},
$$

where

$$
d_{\kappa}(w)=d_{\kappa}(w, P)=\operatorname{ord}_{w}(P)-\min \left\{\operatorname{ord}_{w}(P), \operatorname{ord}_{w+\kappa}(P)\right\},
$$

with $\operatorname{ord}_{w}(P) \geq 0$ being the order of zero of the polynomial $P$ at $w \in \mathbb{C}$. We note here even if $\kappa=1$, the definition of $\kappa$-difference radical is not the same as our definition above. For example, if $g(z)=z^{2}(z-1)(z-2)$, then $\operatorname{rãd}_{1}(g)=z(z-2)$, which is different from $\operatorname{rad}_{\Delta}(g)=z^{2}$. On the other hand, the ideas in [4] work for considering some problems even though the definitions are different. Actually, there are several properties in common. For the sake of simplicity, we write $d_{1}(w)$ given in (3.4) as $d(w)$, and write $\operatorname{rãd}_{1}(P)$ given in (3.3) as rãd $(P)$. We have the following proposition.

Proposition 3.1 Let $P$ be a polynomial. Then $\operatorname{deg} \operatorname{rad}_{\Delta}(P)=\operatorname{deg} \operatorname{rãd}(P)$.

Proof. When $P$ is a constant, the assertion holds. We assume that $P$ is a nonconstant polynomial. Let $Z_{P}$ be the set of zeros of $P$, i.e., $Z_{P}=\{w \mid P(w)=0\}$ considering the multiplicities. In other words, if $w$ is a zero of $P$ then $w$ appears $\operatorname{ord}_{w}(P)$ times in $Z_{P}$.

Firstly we consider deg $\operatorname{rad}_{\Delta}(P)$. The process to determine (3.1) is translated as follows. Choose $z_{1} \in Z_{P}$ satisfying $z_{1}-1 \notin Z_{P}$. If $z_{1}+1 \in Z_{P}$ then we adopt $z_{1}+1$. Repeating this procedure, we adopt $z_{1}+k \in Z_{P}, k \in \mathbb{N}$ and stop $z_{1}+n_{1}-1 \in Z_{P}$ when $z_{1}+n_{1} \notin Z_{P}$. Then we find a finite sequence $L_{1}=\left\{z_{1}, z_{1}+1, \ldots, z_{1}+n_{1}-1\right\}$. We may have $L_{1}=\left\{z_{1}\right\}$ in case $z_{1}+1 \notin Z_{P}$. Next Choose $z_{2} \in Z_{P} \backslash L_{1}$ satisfying $z_{2}-1 \notin Z_{P} \backslash L_{1}$. It may be possible $z_{1}=z_{2}$ when $z_{1}$ is a zero of $P$ with multiplicity $\geq 2$. If $z_{2}+1 \in Z_{P} \backslash L_{1}$ then we adopt $z_{2}+1$. Repeating this procedure, we adopt $z_{2}+k \in Z_{P} \backslash L_{1}, k \in \mathbb{N}$ and stop $z_{2}+n_{2}-1 \in Z_{P} \backslash L_{1}$ when $z_{2}+n_{2} \notin Z_{P} \backslash L_{1}$. Then we find a finite sequence 
$L_{2}=\left\{z_{2}, z_{2}+1, \ldots, z_{2}+n_{2}-1\right\}$. Continuing this process, we divide $Z_{P}$ into $N \in \mathbb{N}$ finite sequences, namely,

$$
Z_{P}=\bigcup_{j=1}^{N} L_{j}=\bigcup_{j=1}^{N}\left\{z_{j}, z_{j}+1, \ldots, z_{j}+n_{j}-1\right\},
$$

with $z_{j}-1, z_{j}+n_{j} \notin Z_{P} \backslash\left(\bigcup_{k=1}^{j-1} L_{k}\right)$ for any $j$. We see that $N$, say the number of $L_{j}$ 's, coincides with $\operatorname{rad}_{\Delta}(P)$.

Secondly we observe deg rãd( $P$ ). By (3.4), we have for $w \in Z_{P}$

$$
d(w)=\operatorname{ord}_{w}(P)-\min \left\{\operatorname{ord}_{w}(P), \operatorname{ord}_{w+1}(P)\right\}=\max \left\{0, \operatorname{ord}_{w}(P)-\operatorname{ord}_{w+1}(P)\right\} .
$$

If $w \in Z_{P}$ satisfies $\operatorname{ord}_{w}(P)-\operatorname{ord}_{w+1}(P)>0$, then $w$ contributes to $\operatorname{rãd}(P)$. Choose $w_{1} \in Z_{P}$ satisfying $w_{1}+1 \notin Z_{P}$, which contributes to $\operatorname{rãd}(P)$. If $w_{1}-1 \in Z_{P}$ then we adopt $w_{1}-1$. Repeating this procedure, we adopt $w_{1}-k \in Z_{P}, k \in \mathbb{N}$ and stop $w_{1}-m_{1}+1 \in Z_{P}$ when $z_{1}-m_{1} \notin Z_{P}$. Then we find a finite sequence $K_{1}=\left\{w_{1}, w_{1}-1, \ldots, w_{1}-m_{1}+1\right\}$. Note that $w_{1}$ can be chosen $\operatorname{ord}_{w_{1}}(P)$ times in the first step in case $\operatorname{ord}_{w_{1}}(P) \geq 2$, and we may have $K_{1}=\left\{w_{1}\right\}$ in case $w_{1}-1 \notin Z_{P}$. Next Choose $w_{2} \in Z_{P} \backslash K_{1}$ satisfying $w_{2}+1 \notin Z_{P} \backslash K_{1}$. It may be possible $w_{1}=w_{2}$ when $w_{1}$ is a zero of $P$ with multiplicity $\geq 2$ as mentioned above. If $w_{2}-1 \in Z_{P} \backslash K_{1}$ then we adopt $w_{2}-1$. Repeating this procedure, we adopt $w_{2}-k, k \in \mathbb{N}$ and stop $w_{2}-m_{2}+1 \in Z_{P} \backslash K_{1}$ when $w_{2}-m_{2} \notin Z_{P} \backslash K_{1}$. Then we find a finite sequence $K_{2}=\left\{w_{2}, w_{2}-1, \ldots, w_{2}-m_{2}+1\right\}$. Continuing this process, we divide $Z_{P}$ into $M \in \mathbb{N}$ finite sequences, namely,

$$
Z_{P}=\bigcup_{j=1}^{M} K_{j}=\bigcup_{j=1}^{M}\left\{w_{j}, w_{j}-1, \ldots, w_{j}-m_{j}+1\right\},
$$

with $w_{j}+1, w_{j}-m_{j} \notin Z_{P} \backslash\left(\bigcup_{k=1}^{j-1} K_{k}\right)$ for any $j$. Then we see that $\operatorname{rãd}(P)=\prod_{j=1}^{M}\left(z-w_{j}\right)=$ $\prod_{w \in \mathbb{C}}(z-w)^{d_{w}}$.

The finite sequences $L_{j} \subset Z_{P}$ and $K_{j} \subset Z_{P}$ possess a common property that outsides of these sequences $z_{j}-1, z_{j}+n_{j}, w_{j}+1, w_{j}-m_{j}$ do not belong to $Z_{P}$. This division of $Z_{P}$ is uniquely determined, which implies $N=M$. This concludes that $\operatorname{deg} \operatorname{rad}_{\Delta}(P)=$ $\operatorname{deg} \operatorname{rãd}(P)$.

Remark 2 Using the notations in the proof of Proposition [3.1, we have

$$
\operatorname{rad}_{\Delta}(P)=\prod_{j=1}^{N}\left(z-z_{j}\right) \quad \text { and } \quad \operatorname{rãd}(P)=\prod_{j=1}^{M}\left(z-w_{j}\right) .
$$

Consider the example $g(z)=z^{2}(z-1)(z-2)$ which is treated just below (3.4). The zeros $\{0,0,1,2\}$ of $g$ are divided into two sequences $\{0\}$ and $\{0,1,2\}$. Thus we confirm that $\operatorname{rad}_{\Delta}(g)=z \cdot z=z^{2}$ and $\operatorname{rãd}(g)=z(z-2)$. We observe another example $h(z)=$ $(z+1) z^{2}(z-1)^{3}(z-2)^{2}(z-4)$. The zeros $\{-1,0,0,1,1,1,2,2,4\}$ of h are divided into four sequences $\{-1,0,1,2\},\{0,1,2\},\{1\}$ and $\{4\}$, which gives that $\operatorname{rad}_{\Delta}(g)=(z+1) z(z-$ 1) $(z-4)$ and $\operatorname{rãd}(g)=(z-1)(z-2)^{2}(z-4)$. 
Suppose that $f$ and $g$ are entire functions, and suppose that $z_{1} \in \mathbb{C}$ is a shifting zero of $f$ with height $m$ and $z_{2} \in \mathbb{C}$ is a shifting zero of $g$ with height $n$. Then from Theorem 2.1, there exist entire functions $F$ and $G$ such that $f(z)=\left(z-z_{1}\right) \frac{m_{1}}{} F(z)$ and $g(z)=\left(z-z_{2}\right) \stackrel{n_{1}}{ } G(z)$, where $1 \leq m_{1} \leq m$ and $1 \leq n_{1} \leq n$. If

$$
f(z) g(z)=\left(z-z_{0}\right) \frac{m_{1}+n_{1}}{2} F(z) G(z)
$$

where $z_{0}$ is $z_{1}$ or $z_{2}$, then $z-z_{0}$ is called the common shifting divisor of $f$ and $g$, which is the analogue of classical common divisor. If $z_{0}=z_{1}$, then $z_{2}=z_{1}+m_{1}$, and if $z_{0}=z_{2}$, then $z_{1}=z_{2}+n_{1}$. For example, if $f(z)=z(z-1)(z-2)$ and $g(z)=(z-2)(z-3)(z-4)$, then $z, z-1, z-2$ are the common shifting divisor of $f$ and $g$. In addition, $z-2$ is the common divisor of $f$ and $g$. If $f$ and $g$ do not have any nonconstant shifting common divisors, then $f$ and $g$ are called shifting prime.

Remark 3 Let $P$ and $Q$ be nonconstant polynomials. In general, we have

$$
\operatorname{deg}\left(\operatorname{rad}_{\Delta}(P Q)\right) \leq \operatorname{deg}\left(\operatorname{rad}_{\Delta}(P)\right)+\operatorname{deg}\left(\operatorname{rad}_{\Delta}(Q)\right) .
$$

By the definition of the shifting prime, the equality in (3.6) holds if $P$ and $Q$ are shifting prime. On the other hand, even though the equality in (3.6) holds, $P$ and $Q$ are not always shifting prime. For example, consider $P(z)=z$ and $Q(z)=z(z-1)$. Then $\operatorname{rad}_{\Delta}(P Q)=$ $z^{2}$, and $\operatorname{rad}_{\Delta}(P)=z, \operatorname{rad}_{\Delta}(Q)=z$, which gives the equality in (3.6) holds. However, $P$ and $Q$ are not shifting prime. In fact, we can write $P(z)=z^{1} \cdot 1, Q(z)=z^{\underline{1}} \cdot(z-1)$ and $P(z) Q(z)=z \underline{1+1} \cdot z$.

In the following, we denote the greatest common divisor of $f$ and $g$ by $\operatorname{gcd}(f, g)$. It is well known that if two entire functions $f$ and $g$ are prime, then $\operatorname{gcd}\left(f, f^{\prime}\right)$ and $\operatorname{gcd}\left(g, g^{\prime}\right)$ are also prime. We state the difference analogue of this result as follows.

Lemma 3.1 Suppose that $f$ and $g$ are entire functions and shifting prime. Then $\operatorname{gcd}(f, \Delta f)$ and $\operatorname{gcd}(g, \Delta g)$ are also shifting prime. Moreover, $\operatorname{gcd}(f, \Delta f)$ and $\operatorname{gcd}(g, \Delta g)$ are prime.

Proof. Assume that $\operatorname{gcd}(f, \Delta f)$ and $\operatorname{gcd}(g, \Delta g)$ have a common shifting divisor. This leads that there exist $z_{1}, z_{2} \in \mathbb{C}, m, n \in \mathbb{N}$ and entire functions $J$ and $K$ such that

$$
\operatorname{gcd}(f, \Delta f)=\left(z-z_{1}\right)^{\underline{m}} J(z)
$$

and

$$
\operatorname{gcd}(g, \Delta g)=\left(z-z_{2}\right)^{\underline{n}} K(z)
$$

We assume that $z_{2}=z_{1}+m$ without loss of generality. Then we have

$$
\operatorname{gcd}(f, \Delta f) \operatorname{gcd}(g, \Delta g)=\left(z-z_{1}\right) \frac{m+n}{} J(z) K(z) .
$$

It follows from (3.7), (3.8) and Corollary 2.2 that $f(z)=\left(z-z_{1}\right) \frac{m+1}{2} J_{1}(z)$ and $g(z)=$ $\left(z-z_{2}\right) \frac{n+1}{2} K_{1}(z)$, where $J_{1}$ and $K_{1}$ are entire functions. In this way, we have

$$
f(z) g(z)=\left(z-z_{1}\right)^{\frac{m+n+1}{2}}\left(z-z_{1}+m\right) J_{1}(z) K_{1}(z) .
$$

It shows that $f$ and $g$ have a common shifting divisor $z-z_{1}$, which is a contradiction with our assumption. We thus proved the first assertion. 
Assume that $\operatorname{gcd}(f, \Delta f)$ and $\operatorname{gcd}(g, \Delta g)$ are not prime. Let $z-z_{0}$ be the common divisor of $\operatorname{gcd}(f, \Delta f)$ and $\operatorname{gcd}(g, \Delta g)$. Then $\operatorname{gcd}(f, \Delta f)=\left(z-z_{0}\right) S(z)$ and $\operatorname{gcd}(g, \Delta g)=$ $\left(z-z_{0}\right) T(z)$, where $S$ and $T$ are entire functions. By Corollary [2.2, we obtain that $f(z)=\left(z-z_{0}\right)^{2} S_{1}(z)$ and $g(z)=\left(z-z_{0}\right)^{2} T_{1}(z)$, where $S_{1}$ and $T_{1}$ are entire functions. It implies that $f$ and $g$ are not shifting prime, which is a contradiction to our assumption. Therefore, we proved Lemma 3.1.

Let $a, b$ and $c$ be relatively prime polynomials such that not all of them are identically zero. If they satisfy $a+b=c$, then we have (1.1) by the Stothers-Mason theorem. For a polynomial $P$, we write $\tilde{n}=\operatorname{deg}(\operatorname{rãd}(P))$, where $\operatorname{rãd}(P)$ is given in (3.3) with $\kappa=1$. The difference analogue of the Stothers-Mason theorem is given in [4, Theorem 3.1], which states that if $a+b=c$, then

$$
\max \{\operatorname{deg}(a), \operatorname{deg}(b), \operatorname{deg}(c)\} \leq \tilde{n}(a)+\tilde{n}(b)+\tilde{n}(c)-1 .
$$

The inequality (3.9) is sharp, see [4, Example 3.2], which is mentioned below in Remark 4, In general, $\tilde{n}(a b c) \leq \tilde{n}(a)+\tilde{n}(b)+\tilde{n}(c)$, and equality holds under some conditions. We here consider the shifting prime condition and state a different version of difference analogue of the Stothers-Mason theorem in terms of $\operatorname{rad}_{\Delta}(P)$ defined by (3.2) as follows.

Theorem 3.2 Let $a, b$ and $c$ be relatively shifting prime polynomials such that

$$
a+b=c
$$

and such that $a, b$ and $c$ are not all constant. Then

$$
\max \{\operatorname{deg}(a), \operatorname{deg}(b), \operatorname{deg}(c)\} \leq \operatorname{deg}\left(\operatorname{rad}_{\Delta}(a b c)\right)-1=\tilde{n}(a b c)-1 .
$$

In order to prove Theorem 3.2, we need the following lemma corresponding to the result [4, Lemma 2.1], which reveals the degree relation between $P$ and $\operatorname{gcd}(P, \Delta P)$ when $P$ is a polynomial.

Lemma 3.3 Let $P$ be a nonzero polynomial. Then

$$
\operatorname{deg}(P)=\operatorname{deg}(\operatorname{gcd}(P, \Delta P))+\operatorname{deg}\left(\operatorname{rad}_{\Delta}(P)\right) .
$$

Proof. By (3.1), $P$ can be written by $P(z)=A \prod_{j=1}^{N}\left(z-z_{j}\right) \frac{n_{j}}{3}$ with a constant $A \neq 0$. It follows from the arguments in Corollary 2.2, $\Delta P$ does not have factor $z-z_{j}+n_{j}-1$, $j=1,2, \ldots, N$. Hence we have

$$
\operatorname{gcd}(P(z), \Delta P(z))=\prod_{j=1}^{m}\left(z-z_{j}\right) \frac{n_{j}-1}{} .
$$

By (3.2) and (3.12), we obtain (3.11). We have thus proved Lemma 3.3 .

We also need to recall a summation property, see e.g., [6, Page 48], [7, Pages 115-116], [12, 327-328].

Lemma 3.4 Let $R(z)$ be a rational function. We write $R(z)$ in the form

$$
R(z)=\rho \frac{\prod_{k=1}^{n}\left(z-\alpha_{k}\right)}{\prod_{j=1}^{m}\left(z-\beta_{j}\right)}
$$


where $\rho \neq 0, \alpha_{k}, k=1, \ldots, n$ and $j=1, \ldots, m$ are complex numbers. The first order linear homogeneous equation

$$
y(z+1)=R(z) y(z)
$$

can be solved as

$$
y(z)=\pi(z) \rho^{z} \frac{\prod_{k=1}^{n} \Gamma\left(z-\alpha_{k}\right)}{\prod_{j=1}^{m} \Gamma\left(z-\beta_{j}\right)}
$$

where $\pi(z)$ is an arbitrary periodic function of period 1 .

Proof of Theorem 3.2. We may suppose that $\operatorname{deg}(c)=\max \{\operatorname{deg}(a), \operatorname{deg}(b), \operatorname{deg}(c)\}$ without loss of generality. Since $a+b=c$, we have $\Delta a+\Delta b=\Delta c$. Multiplying the first equation by $\Delta a$, the second by $a$, and then subtracting them, we obtain

$$
b \Delta a-a \Delta b=c \Delta a-a \Delta c .
$$

We see that $\operatorname{gcd}(a, \Delta a), \operatorname{gcd}(b, \Delta b), \operatorname{gcd}(c, \Delta c)$ all divide $b \Delta a-a \Delta b$ by (3.14). Since $a, b$ and $c$ are relatively shifting prime, from Lemma $3.1 \operatorname{gcd}(a, \Delta a), \operatorname{gcd}(b, \Delta b), \operatorname{gcd}(c, \Delta c)$ are relatively prime. It yields that they are the factors of $b \Delta a-a \Delta b$. Then we have

$$
\operatorname{deg}(\operatorname{gcd}(a, \Delta a))+\operatorname{deg}(\operatorname{gcd}(b, \Delta b))+\operatorname{deg}(\operatorname{gcd}(c, \Delta c)) \leq \operatorname{deg}(a)+\operatorname{deg}(b)-1
$$

provided that $b \Delta a-a \Delta b \neq 0$. We add $\operatorname{deg}(c)$ to both sides, and we apply Lemma 3.3,

$$
\begin{aligned}
\operatorname{deg}(c) \leq & \operatorname{deg}(a)-\operatorname{deg}(\operatorname{gcd}(a, \Delta a))+\operatorname{deg}(b)-\operatorname{deg}(\operatorname{gcd}(b, \Delta b)) \\
& +\operatorname{deg}(c)-\operatorname{deg}(\operatorname{gcd}(c, \Delta c))-1 \\
\leq & \operatorname{deg}\left(\operatorname{rad}_{\Delta}(a)\right)+\operatorname{deg}\left(\operatorname{rad}_{\Delta}(b)\right)+\operatorname{deg}\left(\operatorname{rad}_{\Delta}(c)\right)-1
\end{aligned}
$$

By means of Remark 3 , we obtain (3.10) when $b \Delta a-a \Delta b \neq 0$.

Below we consider the case when $b \Delta a-a \Delta b=0$. From (3.14),

$$
\frac{\Delta a}{a}=\frac{\Delta b}{b}=\frac{\Delta c}{c}=\kappa(z),
$$

where $\kappa(z)$ is a rational function. Then polynomials $a, b$ and $c$ are solutions of the first order linear difference equation $\Delta f(z)=\kappa(z) f(z)$, namely

$$
f(z+1)=(\kappa(z)+1) f(z) .
$$

By means of Lemma 3.4, there exists a nonconstant polynomial $p$ so that $a=\pi_{1} p, b=\pi_{2} p$ and $c=\pi_{3} p$, where $\pi_{j}, j=1,2,3$ are periodic functions with period 1. Since a periodic function is a transcendental function or a constant, the only possibility is that $\pi_{j}, j=1,2,3$ are all constants. We claim that every shifting zero of $p$ is simple height. Otherwise, $a, b$ and $c$ are not relatively shifting prime. Hence, we have

$$
\operatorname{deg}\left(\operatorname{rad}_{\Delta}(a b c)\right)=\operatorname{deg}(a)+\operatorname{deg}(b)+\operatorname{deg}(c) .
$$

It is easy to see that inequality in (3.10) holds in this case. The right equality follows from Proposition 3.1. We have thus proved Theorem 3.2

Remark 4 We could see the assertion of Theorem 3.2 is sharp. For example, let $a(z)=$ $z(z-1), b(z)=-(z-4)(z-5)$ and $c(z)=4(2 z-5)$. We see that $a, b$ and $c$ are relatively shifting prime polynomials satisfying $a+b=c$. In addition, it is shown that $\max \{\operatorname{deg}(a), \operatorname{deg}(b), \operatorname{deg}(c)\}=2, \operatorname{rad}_{\Delta}(a b c)=z(z-5 / 2)(z-4)$, and $\operatorname{deg}\left(\operatorname{rad}_{\Delta}(a b c)\right)=3$. 


\section{Extension of the Stothers-Mason theorem with dif- ference radical}

We write $P$ in the form (3.1), i.e., $P(z)=A \prod_{j=1}^{N}\left(z-z_{j}\right)$ 蕩. The difference radical of truncation level $q \in \mathbb{N}$ for a polynomial $P(z)$ which is denoted by

$$
\operatorname{rad}_{\Delta}^{q}(P)=\operatorname{gcd}\left(\prod_{j=1}^{N}\left(z-z_{j}\right) \frac{n_{j}}{}, \prod_{j=1}^{N}\left(z-z_{j}\right) \underline{q}\right) .
$$

It is a generalization of difference radical. When $q=1$ in (4.1), it reduces to the difference radical of $P$ given in (3.2). We note that $\operatorname{rad}_{\Delta}^{q}(P)=\operatorname{rad}_{\Delta}(P)$ holds for any $q \geq 2$ if and only if all the shifting zeros of $P$ are simple height. It follows from (4.1),

$$
\operatorname{deg}\left(\operatorname{rad}_{\Delta}^{q}(P)\right)=\operatorname{deg}\left(\prod_{j=1}^{N}\left(z-z_{j}\right) \frac{\min \left(n_{j}, q\right)}{}\right)=\sum_{j=1}^{N} \min \left(n_{j}, q\right) .
$$

In addition, we have an inequality

$$
\operatorname{deg}\left(\operatorname{rad}_{\Delta}^{q}(P)\right) \leq q \cdot \operatorname{deg}\left(\operatorname{rad}_{\Delta}(P)\right) .
$$

The following theorem extends Theorem 3.2 for $m+1$ polynomials, where $m \in \mathbb{N}$, $m \geq 2$. We state it as follows.

Theorem 4.1 Let $m \in \mathbb{N}, m \geq 2$ and let $f_{1}, \ldots, f_{m+1}$ be pairwise relatively shifting prime polynomials with $\min _{1 \leq i \leq m+1} \operatorname{deg} f_{i} \geq m-1$ satisfying the following functional equation

$$
f_{1}+\cdots+f_{m}=f_{m+1},
$$

and such that $f_{1}, \ldots, f_{m}$ are linearly independent over $\mathbb{C}$. Then

$$
\begin{aligned}
\max _{1 \leq i \leq m+1}\left\{\operatorname{deg} f_{i}\right\} & \leq \operatorname{deg}\left(\operatorname{rad}_{\Delta}^{m-1}\left(f_{1} f_{2} \cdots f_{m+1}\right)\right)-\frac{1}{2} m(m-1) \\
& \leq(m-1) \operatorname{deg}\left(\operatorname{rad}_{\Delta}\left(f_{1} f_{2} \cdots f_{m+1}\right)\right)-\frac{1}{2} m(m-1) .
\end{aligned}
$$

In order to prove Theorem 4.1, we need two lemmas below. We first consider a generalization of Lemma 3.3 . For $x \in \mathbb{R}$, we write $[x]^{+}=\max \{x, 0\}$.

Lemma 4.2 Let $n \in \mathbb{N}$ and let $P$ be a polynomial with $\operatorname{deg} P \geq n$ represented by (3.1). Then

$$
\operatorname{deg}\left(\operatorname{gcd}\left(P, \Delta P, \ldots, \Delta^{n} P\right)\right)=\sum_{j=1}^{N}\left[n_{j}-n\right]^{+}
$$

and

$$
\operatorname{deg}(P)-\operatorname{deg}\left(\operatorname{gcd}\left(P, \Delta P, \ldots, \Delta^{n} P\right)\right)=\operatorname{deg}\left(\operatorname{rad}_{\Delta}^{n}(P)\right)
$$

Proof. Let $z_{j}$ be a shifting zero of $P$ with height $n_{j}$ such that $P\left(z_{j}-1\right) \neq 0$. We write $P(z)=\left(z-z_{j}\right) \frac{n_{j}}{\underline{ }} G_{j}(z)$, where $G_{j}$ is a polynomial. From the arguments in the proof of Corollary 2.2, $\Delta P(z)=\left(z-z_{j}\right) \frac{n_{j}-1}{{ }_{2}} Q_{j, 1}(z)$, where $Q_{j, 1}$ is a polynomial satisfying $Q_{j, 1}\left(z_{j}+\right.$

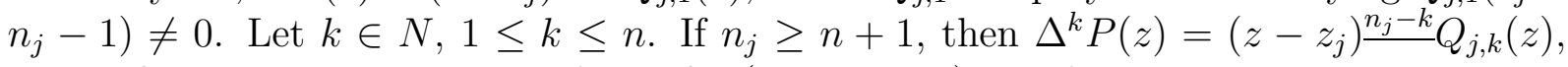
where $Q_{j, k}$ is a polynomial satisfying $Q_{j, k}\left(z_{j}+n_{j}-k\right)=0$ for any $1 \leq k \leq n-1$. This 
implies that $\operatorname{gcd}\left(P, \Delta P, \ldots, \Delta^{n} P\right)$ is divided by $\left(z-z_{j}\right) \frac{n_{j}-n}{}$. If $n_{j} \leq n$, then the factors in $\left(z-z_{j}\right) \underline{n_{j}}$ never contribute to $\operatorname{gcd}\left(P, \Delta P, \ldots, \Delta^{n} P\right)$. Then

$$
\operatorname{gcd}\left(P, \Delta P, \ldots, \Delta^{n} P\right)=\prod_{j=1}^{N}\left(z-z_{j}\right) \frac{\left[n_{j}-n\right]^{+}}{}
$$

which implies (4.6). From (4.6), (3.1) and (4.2), we obtain (4.7). In fact,

$$
\begin{gathered}
\operatorname{deg}\left(\operatorname{gcd}\left(P, \Delta P, \ldots, \Delta^{n} P\right)\right)=\sum_{j=1}^{N}\left[n_{j}-n\right]^{+}=\sum_{j=1}^{N} \max \left(n_{j}-n, 0\right) \\
=\sum_{j=1}^{N} n_{j}-\sum_{j=1}^{N} \min \left(n_{j}, n\right)=\operatorname{deg}(P)-\operatorname{deg}\left(\operatorname{rad}_{\Delta}^{n}(P)\right) .
\end{gathered}
$$

We have thus proved Lemma 4.2 .

Remark 5 Let $n \in \mathbb{N}$ and let $P$ and $Q$ be nonconstant polynomials. We have the following estimate as well as Remark 3 .

$$
\operatorname{deg}\left(\operatorname{rad}_{\Delta}^{n}(P Q)\right) \leq \operatorname{deg}\left(\operatorname{rad}_{\Delta}^{n}(P)\right)+\left(\operatorname{rad}_{\Delta}^{n}(Q)\right)
$$

By the definition of the shifting prime, the equality in (3.6) holds if $P$ and $Q$ are shifting prime.

Let $m \in \mathbb{N}$ and let $f_{i}(z), 1 \leq i \leq m$ be polynomials. We next recall the definition of the Casoratian (Casorati determinant), which has played important roles in difference calculus, see e.g., [12, Pages 354-357], [14, Pages 276-281].

$$
\mathcal{C}(z)=\mathcal{C}\left(f_{1}, f_{2}, \ldots, f_{m}\right)=\left|\begin{array}{cccc}
f_{1}(z) & f_{2}(z) & \cdots & f_{m}(z) \\
\Delta f_{1}(z) & \Delta f_{2}(z) & \cdots & \Delta f_{m}(z) \\
& \ldots \ldots \ldots \cdots \cdots & \\
& \ldots \ldots \ldots & \\
\Delta^{m-1} f_{1}(z) & \Delta^{m-1} f_{2}(z) & \cdots & \Delta^{m-1} f_{m}(z)
\end{array}\right|
$$

We need a number of known results in linear algebra e.g., 8]. By the properties of the determinant, we have

Lemma 4.3 The following claims hold.

(i) It is possible to replace $\Delta^{k} f_{i}$ with $f_{i}(z+k), i=1,2, \ldots, m, k=1,2, \ldots, m-1$ in $\mathcal{C}(z)$.

(ii) $\mathcal{C}(z)$ does not vanish identically in case $f_{i}(z), 1 \leq i \leq m$ are linearly independent over $\mathbb{C}$.

(iii) It is possible to replace $f_{i}$ with $f_{m+1}=f_{1}+f_{2}+\cdots+f_{m}$ for arbitrary fixed $i \in$ $\{1,2, \ldots, m\}$ in $\mathcal{C}(z)$.

(iv) $\mathcal{C}(z)$ is divided by $\left.\operatorname{gcd}\left(f_{i}, \Delta f_{i}, \ldots, \Delta^{m-1} f_{i}\right)\right), i=1,2, \ldots, m$, some of which may be constants. 
The equation (4.4) was considered in [4, Theorem 3.5] by the estimates of degree of Casoratian $\mathcal{C}(z)$ replacing $\Delta^{k} f_{i}$ with $f_{i}(z+k), i=1,2, \ldots, m, k=1,2, \ldots, m-1$ in $\mathcal{C}(z)$, see Lemma 4.3 (i). Following the idea in [4], we prove Theorem 4.1.

Proof of Theorem 4.1 We consider the Casoratian $\mathcal{C}(z)$ of $f_{1}(z), \ldots, f_{m}(z)$ defined by (4.10). Since $f_{1}, \ldots, f_{m}$ are linearly independent over $\mathbb{C}$, we have $\mathcal{C}(z) \not \equiv 0$ by Lemma 4.3 (ii).

By (4.4) and Lemma 4.3 (iii), we can replace $f_{i}$ with $f_{m+1}$ for arbitrary fixed $i \in$ $\{1,2, \ldots, m\}$ in the right hand of (4.10).

Write $f_{i}(z)=A_{i} \prod_{j=1}^{N_{i}}\left(z-z_{i, j}\right) \frac{n_{i, j}}{}, f_{i}\left(z_{i, j}-1\right) \neq 0,1 \leq j \leq N_{i}, 1 \leq i \leq m+1$ following (3.1), where $A_{i}$ are constants, $N_{i}, n_{i, j} \in \mathbb{N}$. This gives that $f_{i}(z)$ is divided by

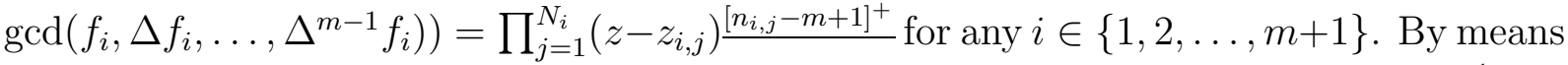
of Lemma 4.3 (iv), we see that the Casoratian $\mathcal{C}(z)$ is divided by $\operatorname{gcd}\left(f_{i}, \Delta f_{i}, \ldots, \Delta^{m-1} f_{i}\right)$ ), $1 \leq i \leq m+1$. Since we assume that $f_{1}, \ldots, f_{m+1}$ are pairwise relatively shifting prime, $\mathcal{C}(z)$ is divided by $q(z):=\prod_{i=1}^{m+1} \prod_{j=1}^{N_{i}}\left(z-z_{i, j}\right) \underline{\left[n_{i, j}-m+1\right]^{+}}$, namely there exists a polynomial $p(z)$ such that $\mathcal{C}(z)=p(z) q(z)$.

By the assumption $\min _{1 \leq i \leq m} \operatorname{deg} f_{i} \geq m-1$, we have $\operatorname{deg}\left(\Delta^{k} f_{i}\right)=\operatorname{deg} f_{i}-k \geq 0$, $0 \leq k \leq m-1$. This implies that $\operatorname{deg} \mathcal{C}(z)$ is never beyond any sum of distinct $m$ of the $\operatorname{deg} f_{i}(z), 1 \leq i \leq m+1$ minus $\sum_{k=0}^{m-1} k=m(m-1) / 2$ as the sum of $\operatorname{deg}\left(\Delta^{k} f_{j_{\nu}}\right)$ for the mutually distinct $m$ integers $j_{\nu} \in\{1, \ldots, m, m+1\}$. Since $m \in \mathbb{N}$ and $f_{1}, \ldots, f_{m+1}$ are pairwise relatively shifting prime, by Lemma 4.2 and Remark 5 we have

$$
\begin{aligned}
\min _{1 \leq h \leq m+1} & \left.\sum_{1 \leq i \leq m+1, i \neq h} \operatorname{deg} f_{i}-\frac{1}{2} m(m-1) \geq \operatorname{deg}\left(\prod_{i=1}^{m+1} \operatorname{gcd}\left(f_{i}, \Delta f_{i}, \ldots, \Delta^{m-1} f_{i}\right)\right)\right) \\
= & \sum_{i=1}^{m+1}\left(\operatorname{deg}\left(f_{i}\right)-\operatorname{deg}\left(\operatorname{rad}_{\Delta}^{m-1}\left(f_{j}\right)\right)\right)=\sum_{i=1}^{m+1} \operatorname{deg}\left(f_{i}\right)-\operatorname{deg}\left(\operatorname{rad}_{\Delta}^{m-1}\left(f_{1} f_{2} \cdots f_{m+1}\right)\right)
\end{aligned}
$$

which implies the first inequality of (4.5). Combining (4.3) and the first inequality of (4.5), we see that the second inequality of (4.5) follows. We have proved Theorem 4.1.

Example 4.1 Let $c \in \mathbb{C} \backslash\{0\}, k \in \mathbb{C} \backslash\{0, \pm 5\}$. Set $\alpha=c+k / 10-1 / 2$ and $\beta=c-$ $k / 10-1 / 2$. We consider polynomials $f_{1}(z)=(z-\alpha)^{\underline{5}}, f_{2}(z)=-(z-\beta)^{\underline{5}}, f_{3}(z)=k(z-c)^{\underline{4}}$, which are are linearly independent over $\mathbb{C}$, and consider a polynomial

$$
f_{4}(z)=-\frac{k(k-5)(k+5)}{50000}\left(1000 z^{2}-(2000 c+3000) z+k^{2}+1000 c^{2}+3000 c+1775\right) .
$$

We see that $f_{1}, f_{2}, f_{3}$ and $f_{4}$ are pairwise relatively shifting prime for some suitable $c$ and $k$, and they satisfy (4.4) of the case $m=3$, i.e., $f_{1}+f_{2}+f_{3}=f_{4}$. We have by definition, $\max _{1 \leq i \leq 4}\left\{\operatorname{deg} f_{i}\right\}=5$, and $\operatorname{deg} \operatorname{rad}_{\Delta}^{2} f_{1}=2, \operatorname{deg} \operatorname{rad}_{\Delta}^{2} f_{2}=2, \operatorname{deg} \operatorname{rad}_{\Delta}^{2} f_{3}=2, \operatorname{deg} \operatorname{rad}_{\Delta}^{2} f_{4}=$ 2 by (4.2). This gives that the first inequality of (4.5) is sharp in Theorem 4.1,

Remark 6 Example 4.1 shows that the inequality in Theorem 3.5 of [4] is sharp for the case when $m=3$.

\section{Polynomial solutions of difference Fermat functional equations}

In this section, we apply Theorem 3.2 and Theorem4.1 to difference Fermat type functional equations for investigating nonexistence of polynomial solutions. We adopt the falling 
expression $P^{\underline{n}}$ instead of $P^{n}$ for a polynomial $P$ in the Fermat type functional equations, which is the same to the case we adopt rising expressions $P^{\bar{n}}$ in some sense. For the Fermat type functional equations, see e.g., [2], [13]. Concerning the methods of the proofs, we follow the idea in [4].

Theorem 5.1 Let $a, b$ and $c$ be polynomials, not all constants, and $n \in \mathbb{N}$ such that $a^{\underline{n}}$, $b^{\underline{n}}$ and $c^{\underline{n}}$ are relatively shifting prime and satisfy

$$
a^{\underline{n}}+b^{\underline{n}}=c^{\underline{n}} .
$$

Then $n \leq 2$. In addition, if one of $a, b$ and $c$ is a constant, then $n=1$.

Proof. Let us first assume that $a, b$ and $c$ are all nonconstant polynomials. By Theorem 3.2 and Remark 3, we have

$$
\begin{aligned}
n \operatorname{deg}(a)=\operatorname{deg}\left(a^{\underline{n}}\right) & \leq \max \left\{\operatorname{deg}\left(a^{\underline{n}}\right), \operatorname{deg}\left(b^{\underline{n}}\right), \operatorname{deg}\left(c^{\underline{n}}\right)\right\} \leq \operatorname{deg}\left(\operatorname{rad}_{\Delta}\left(a^{\underline{n}} b^{\underline{n}} c^{\underline{n}}\right)\right)-1 \\
& \leq \operatorname{deg}\left(\operatorname{rad}_{\Delta}\left(a^{\underline{\underline{n}}}\right)\right)+\operatorname{deg}\left(\operatorname{rad}_{\Delta}\left(b^{\underline{n}}\right)\right)+\operatorname{deg}\left(\operatorname{rad}_{\Delta}\left(c^{\underline{\underline{n}}}\right)\right)-1 \\
& \leq \operatorname{deg}(a)+\operatorname{deg}(b)+\operatorname{deg}(c)-1 .
\end{aligned}
$$

Similarly, for $b$ and $c$, we have

$$
n \operatorname{deg}(b) \leq \operatorname{deg}(a)+\operatorname{deg}(b)+\operatorname{deg}(c)-1
$$

and

$$
n \operatorname{deg}(c) \leq \operatorname{deg}(a)+\operatorname{deg}(b)+\operatorname{deg}(c)-1 .
$$

Combining inequalities (5.2), (5.3) and (5.4), we obtain

$$
n(\operatorname{deg}(a)+\operatorname{deg}(b)+\operatorname{deg}(c)) \leq 3(\operatorname{deg}(a)+\operatorname{deg}(b)+\operatorname{deg}(c))-3,
$$

which implies that $n \leq 2$.

We secondly assume one of $a, b$ and $c$ is a constant. Without loss of generality, we assume that $c$ is a constant. Then (5.2) and (5.3) yield $n(\operatorname{deg}(a)+\operatorname{deg}(b)) \leq 2(\operatorname{deg}(a)+$ $\operatorname{deg}(b))-2$, which shows that $n \leq 1$. We proved our assertion.

We have an example for the case $n=2$, see [4, Example 4.2].

Example 5.1 We consider polynomials $a(z)=z^{2}, b(z)=-(i / 2)\left(\sqrt{2} z^{2}+2 z-\sqrt{2}\right)$ and $c(z)=-(1 / 2)\left(\sqrt{2} z^{2}-2 z-\sqrt{2}\right)$. These polynomials satisfy (5.1). The zeros of $b$ and $c$ are $\{-(1+\sqrt{3}) / \sqrt{2},(-1+\sqrt{3}) / \sqrt{2}\}$ and $\{(1-\sqrt{3}) / \sqrt{2},(1+\sqrt{3}) / \sqrt{2}\}$, respectively. Hence they are relatively shifting prime.

The next result extends Theorem 5.1 to equations with arbitrarily many terms.

Theorem 5.2 Let $m \in \mathbb{N}, m \geq 2, n \in \mathbb{N}$. Suppose that there exist $f_{1}, \ldots, f_{m+1}$ nonconstant polynomials satisfying

$$
f_{1}^{\frac{n}{1}}+f_{2}^{\frac{n}{2}}+\cdots+f_{\frac{n}{m}}=f_{m+1}^{\frac{n}{m}} .
$$

Further suppose that $f_{\frac{n}{1}}^{n}, \ldots, f_{m+1}^{n}$ are pairwise relatively shifting prime and $f_{1}^{n}, \ldots, f \frac{n}{m}$ are linearly independent. Then

$$
n \leq m^{2}-1-\frac{m(m-1)}{2 \max _{1 \leq i \leq m+1}\left\{\operatorname{deg}\left(f_{i}\right)\right\}} .
$$


Proof. By using Theorem 4.1, we have

$$
\begin{aligned}
n \max _{1 \leq i \leq m+1}\left\{\operatorname{deg}\left(f_{i}\right)\right\} & \leq(m-1) \operatorname{deg}\left(\operatorname{rad}_{\Delta}\left(f \frac{n}{1} f \frac{n}{2} \cdots f \frac{n}{m}+1\right)-\frac{1}{2} m(m-1)\right. \\
& \leq(m+1)(m-1) \max _{1 \leq i \leq m+1}\left\{\operatorname{deg}\left(f_{i}\right)\right\}-\frac{1}{2} m(m-1) .
\end{aligned}
$$

Since $\max _{1 \leq i \leq m+1}\left\{\operatorname{deg}\left(f_{i}\right)\right\} \geq 1$, we obtain our assertion.

Remark 7 We change the assumption that $f_{m+1}(z)$ is a nonconstant polynomial in Theorem 5.2 into $f_{m+1}(z) \equiv 1$, and keep other conditions. We use the similar arguments in the proof of Theorem 5.2 with $\operatorname{deg}\left(f_{m+1}\right)=0$. Then we obtain an estimate for polynomial solutions of $f_{1}^{\underline{n}}+f_{\frac{n}{2}}^{n}+\cdots+f_{\frac{n}{m}}=1$,

$$
n \max _{1 \leq i \leq m}\left\{\operatorname{deg}\left(f_{i}\right)\right\} \leq m(m-1) \max _{1 \leq i \leq m}\left\{\operatorname{deg}\left(f_{i}\right)\right\}-\frac{1}{2} m(m-1),
$$

and hence $n \leq m(m-1)\left(1-1 /\left(2 \max _{1 \leq i \leq m}\left\{\operatorname{deg}\left(f_{i}\right)\right\}\right)\right)$. This implies that $n \leq m^{2}-$ $m-1$, which corresponds to the result on polynomial solutions of $f_{1}^{n}+f_{2}^{n}+\cdots+f_{m}^{n}=1$, see e.g., [2], 3]. When $m=3$, we have $n \leq 5$. There exist examples for the case $n=2$ and $n=3$ below.

Example 5.2 Consider relatively shifting prime polynomials $f_{1}(z)=(1 / \sqrt{2}) z+1, f_{2}(z)=$ $(1 / 2) z+(1 / 2)(\sqrt{2}-\sqrt{6})$ and $f_{3}(z)=(\sqrt{3} i / 2) z+(i / 2)(\sqrt{6}-\sqrt{2})$. By computations, we see that $f_{1}, f_{2}$ and $f_{3}$ satisfy

$$
f_{1}^{\frac{2}{1}}+f_{\frac{2}{2}}^{\frac{2}{2}}+f_{\frac{2}{3}}^{2}=1
$$

We consider another triad of relatively shifting prime polynomials $f_{1}(z)=(1 / 24 \sqrt{2})\left(24 z^{2}+\right.$ $48 z-29), f_{2}(z)=(1 / 48)\left(24 z^{2}-48 z-61\right)$ and $f_{3}(z)=(i / 16 \sqrt{3})\left(24 z^{2}+16 z+3\right)$. These polynomials satisfy (5.7).

Example 5.3 Let $s \in \mathbb{C}$ be a root of $s^{9}-144 s^{3}+108=0$ and $t \in \mathbb{C} \backslash\{0\}$. We consider polynomials $f_{1}(z)=z^{3}-a_{2} z^{2}-a_{1} z+a_{0}, f_{2}(z)=-z^{3}+a_{2} z^{2}+a_{1} z-\left(3 a_{0}+s^{3}\right) / 3$ and $f_{3}(z)=s z^{2}+t z+\left(t^{2}-4 s^{2}\right) / 4 s$, where

$$
a_{2}=-\frac{3 t}{2 s}, a_{1}=\frac{3\left(4 s^{2}-t^{2}\right)}{4 s^{2}}, a_{0}=\frac{3 t^{3}-36 s^{2} t-4 s^{6}}{24 s^{3}}
$$

By computations, we see that $f_{1}, f_{2}$ and $f_{3}$ satisfy

$$
f_{1}^{3}+f_{2}^{\frac{3}{2}}+f^{\frac{3}{3}}=1
$$

for an arbitrary $t$. We thus obtain $f_{1}, f_{2}$ and $f_{3}$ that are relatively shifting prime polynomials for a suitable $t$.

\section{References}

[1] Boole, G. A treatise on the calculus of finite differences. Reprint of the 1860 original. Cambridge Library Collection. Cambridge University Press, Cambridge, 2009.

[2] Gundersen, G. G. and W. K. Hayman, The strength of Cartan's version of Nevanlinna theory, Bull. London Math. Soc. 36 (2004), 433-454. 
[3] Gundersen G. G., K. Ishizaki and N. Kimura, Restrictions on meromorphic solutions of Fermat type equations, Proc. Edinburgh Math. Soc. 63 (2020), 654-665.

[4] Ishizaki, K., R. Korhonen, N. Li and K. Toghe, A Stothers-Mason theorem with a difference radical, to appear in Math. Z., https://doi.org/10.1007/s00209-020-026047 .

[5] Ishizaki, K. and Z.-T. Wen, Binomial series and complex difference equations, to appear in J. Math. Anal. Appl., https://doi.org/10.1016/j.jmaa.2020.124844.

[6] Kelley, W. G. and A. C. Peterson, Difference equations. An introduction with applications. Second edition. Harcourt/Academic Press, San Diego, CA, 2001.

[7] Kohno, M., Global analysis in linear differential equations, Mathematics and its Applications, 471, Kluwer Academic Publishers, Dordrecht, 1999.

[8] Lang, S., Introduction to Linear Algebra, Second ed., Springer-Verlag, New York 1988.

[9] Li, N., On the existence of solutions of a Fermat-type difference equation, Ann. Acad. Sci. Fenn. Math. 40 (2015), no. 2, 907-921.

[10] Mason R. C., Diophantine equations over function fields, London Mathematical Society Lecture Note Series, vol. 96, Cambridge University Press, Cambridge, 1984.

[11] Mason, R. C., Norm form equations. I, J. Number Theory 22 (1986), no. 2, 190-207.

[12] Milne-Thomson, L. M., The Calculus of Finite Differences, Macmillan, London, 1933.

[13] Newman, D. J. and M. Slater, Waring's problem for the ring of polynomials, J. Number Theory 11 (1979), 477-487.

[14] Nörlund, N. E., Vorlesungen Über Differenzenrechnung, Springerg Berlin, 1924.

[15] Snyder, N., An alternate proof of Mason's theorem, Elem. Math. 55 (2000), no. 3, 93-94.

[16] Stothers, W. W., Polynomial identities and Hauptmoduln, Quart. J. Math. Oxford Ser. (2) 32 (1981), no. 127, 349-370.

[17] Whittaker, E. T. and G. M. Watson, A course of modern analysis, Cambridge Univ. Press, Cambridge, 1927.

Katsuya Ishizaki

The Open University of Japan, 2-11 Wakaba,

Minama-KU, ChiBA, 261- 8586 JAPAN

email:ishizaki@ouj.ac.jp

Z.-T. Wen

Shantou University, Department of Mathematics, Daxue Road No. 243, Shantou 515063, China

e-mail:zhtwen@stu.edu.cn 